\title{
Ongoing Development of Ultrafast DUV Pulse Laser Repair for EUV Photomasks
}

\author{
Tod Robinson $^{* a}$, Jeff LeClaire ${ }^{\mathrm{a}}$, Iacopo Mochi ${ }^{\mathrm{b}}$, Ricarda Maria Nebling ${ }^{\mathrm{b}}$, Yasin Ekinci ${ }^{\mathrm{b}}$, \\ Dimitrios Kazazis ${ }^{b}$ \\ ${ }^{a}$ Bruker RMR (United States), 430 S. Congress Ave., Ste. 7, Delray Beach, FL, USA 33445; ${ }^{\text {Paul }}$ \\ Scherrer Institute (Switzerland) ODRA/119, Forschungsstrasse 111, 5232 Villigen PSI, Schweiz
}

\begin{abstract}
In prior work, progress was shown in the systematic characterization of the process space for efficient and effective repair of extreme ultraviolet (EUV) photomasks using an ultrafast (femtosecond) pulsed deep ultraviolet (DUV) laser apparatus. In this work, the full analysis and conclusions, along with any additional test results are shown. This includes an analysis of the impact of laser repair on the phase shift of the multilayer using multiple processes.
\end{abstract}

\section{INTRODUCTION}

\subsection{Background}

In a prior paper, it was shown that the laser spot process was able to remove high aspect carbon pindot defects (left panel, Figure 1), but almost no removal was shown for low aspect pindots of the same type. ${ }^{1,2}$ Atomic force microscope (AFM) measured pre and post laser repair defect heights (and full width half max diameters) were analyzed in distinct regimes of defect aspect and laser parameters. There was no observed removal enhancement for carbon defects with $\leq 0.02$ aspect ratio (defect max height divided by its full width half max measure or FWHM).

A novel process was shown which was efficient at rapid, and enhanced over the surrounding pattern, removal of lowaspect carbon or organics (refer right panel of Figure 1). ${ }^{1}$ This dry surface clean process on the laser repair tool was shown to be complimentary with the laser process with enhancement on high-aspect particle (or pindot) repair and/or clean process. This process did significantly reduce high aspect $C$ pindots on EUV sample with no detectable impact to EUV printability (amplitude or phase) although this process did not appear as effective and efficient as it does with low aspect carbon contaminates.

Both of these processes have been successfully applied in customer demo (not all results could be shown) on an optical photomask, and the complementary application of these processes removed all of the defects and contaminates provided. This approach allowed for a more complete EUV photomask repair/clean solution for a femtosecond laser system and has been shown to improve EUV-actinic printability. There was, however, a very large dataset that had yet to be analyzed at the time of the prior publication. A further examination of this dataset is reviewed in this work, especially as pertains to the impact on EUV-actinic phase shift with some more directly quantified measures of EUV print amplitudes as well.

*tod.robinson@bruker.com; phone 1561 330-0411; fax 1561 330-0896; bruker.com

Photomask Japan 2021: XXVII Symposium on Photomask and Next-Generation Lithography Mask Technology, edited by Akihiko Ando, Proc. of SPIE Vol. 11908, 119080O - (C) 2021 SPIE · CCC code: 0277-786X/21/\$21 - doi: 10.1117/12.2601395 


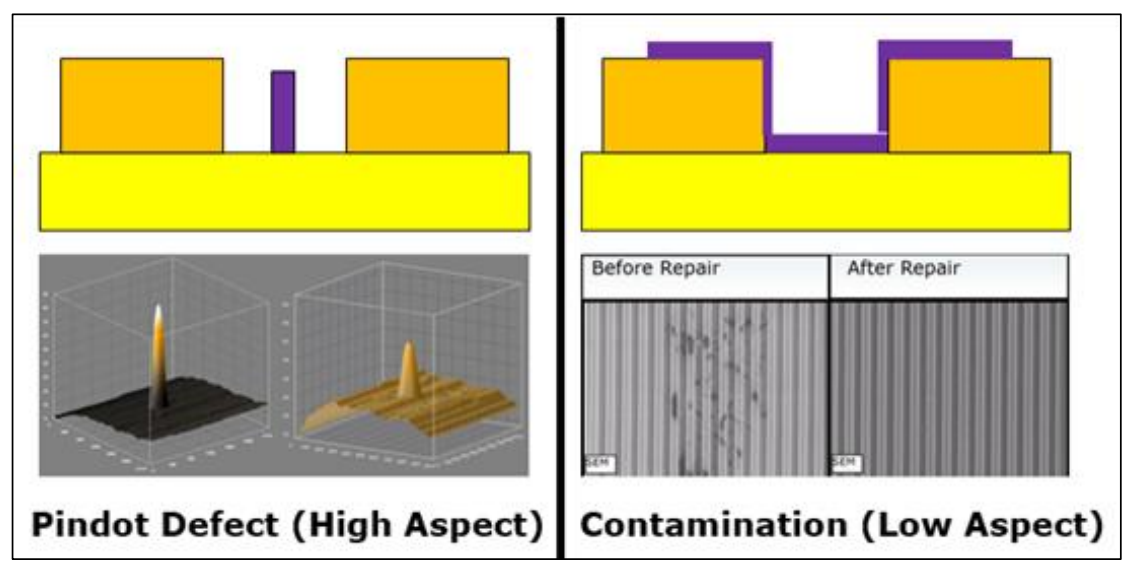

Figure 1, types of surface contamination that may occur on a photomask.

\section{METHODOLOGY}

\subsection{Apparatus and overall test procedure}

The apparatus for this work is summarized in Table 1. The first step of the overall process was to produce a test sample (and adaptor chucks to allow the sample to be loaded in mask repair toolsets) designed specifically for systematic laser repair process testing. A standard test sample was produced by PSI that consisted of repeating cells of different pin dot type absorber defects on multilayer (see Figure 3). This sample was then to be inspected with the RESCAN apparatus for EUV printability of the defects before laser repair and then shipped to Bruker RMR for AFM inspection before laser repair. After these AFM scans, laser repairs are performed on each of the pin dot defects in the targeted test die using varying laser parameters determined from pre-planned full factorial design of experiments. Once these test repairs are performed, the same sites were AFM scanned to determine the topographic changes (if any) of each of these defects. In the final stage, the sample was shipped back to PSI where it was once again inspected with the RESCAN apparatus for EUV printability. Other dies were also inspected for additional (originally unplanned) tests as well as to provide a supplement of pre repair defect data (there were indications in AFM scans that defect variations were similar for the same sub die location).

Table 1, summary of the apparatus used in this work

\begin{tabular}{|c|c|}
\hline Purpose & Apparatus \\
\hline Laser Photomask Repair & Bruker RMR fp-III \\
\hline $\begin{array}{c}\text { EUV Printability } \\
\text { Inspection }\end{array}$ & PSI RESCAN \\
\hline AFM Inspection & Bruker RMR Merlin Nanomachining Tool \\
\hline
\end{tabular}




\subsection{Apparatus review: RESCAN EUV metrology}

RESCAN is a lensless microscope dedicated to EUV mask inspection operating at the Swiss Light Source synchrotron. ${ }^{3}$ The microscope relies on coherent diffraction imaging (CDI) to reconstruct the complex amplitude of the mask surface. A coherent EUV beam is focused on the sample surface with an angle of incidence of 6 degrees. The sample plane is scanned, and a pixel detector records the diffracted light for each position of the object. The complex amplitude of the sample is reconstructed using ptychography ${ }^{4}$, a noise-robust CDI technique that makes it possible to retrieve the phase and the amplitude of the object under investigation. RESCAN can detect amplitude defects as small as 50x50 nm2 and characterize the phase of buried structures with an uncertainty of $2 \% .^{5}$ In the current experiment, each of the reconstructed images has a pixel size of $32.5 \mathrm{~nm}$ and a spatial resolution of $45 \mathrm{~nm}$. Each corresponding dataset is constituted by 141 diffraction patterns, covering a sample area of about $450 \mu \mathrm{m}^{2}$.

\subsection{Apparatus review: fp-III Femtosecond Laser Photomask Repair System}

The fp-III photomask repair tool employs a deep ultraviolet (DUV) $258 \mathrm{~nm}$ wavelength, femtosecond pulsed laser with high numerical aperture (NA) objectives. Compared to prior toolsets, the fp-III has increased stability, reliability, and a smaller cleanroom footprint. Repairs are performed with one of two different objective lenses. The 50X objective is most often used for through-pellicle repairs and dry cleaning (due to its longer working distance) and typically shows thin film removal beam burn diameter around $300 \mathrm{~nm}$ in diameter. The $150 \mathrm{X}$, or highest magnification objective, is used when there is no pellicle mounted on the mask (this is automatically detected at mask load, and if a pellicle is present, this objective is locked out or disabled from being selected). The nominal beam burn diameter for the $150 \mathrm{X}$ on most photomask absorbers is approximately $200 \mathrm{~nm}$. Another unique feature of the fp-III is that it has integrated features to support particle and surface clean processes that allow for EUV photomask defect selective repair and clean with no detectable mask damage.

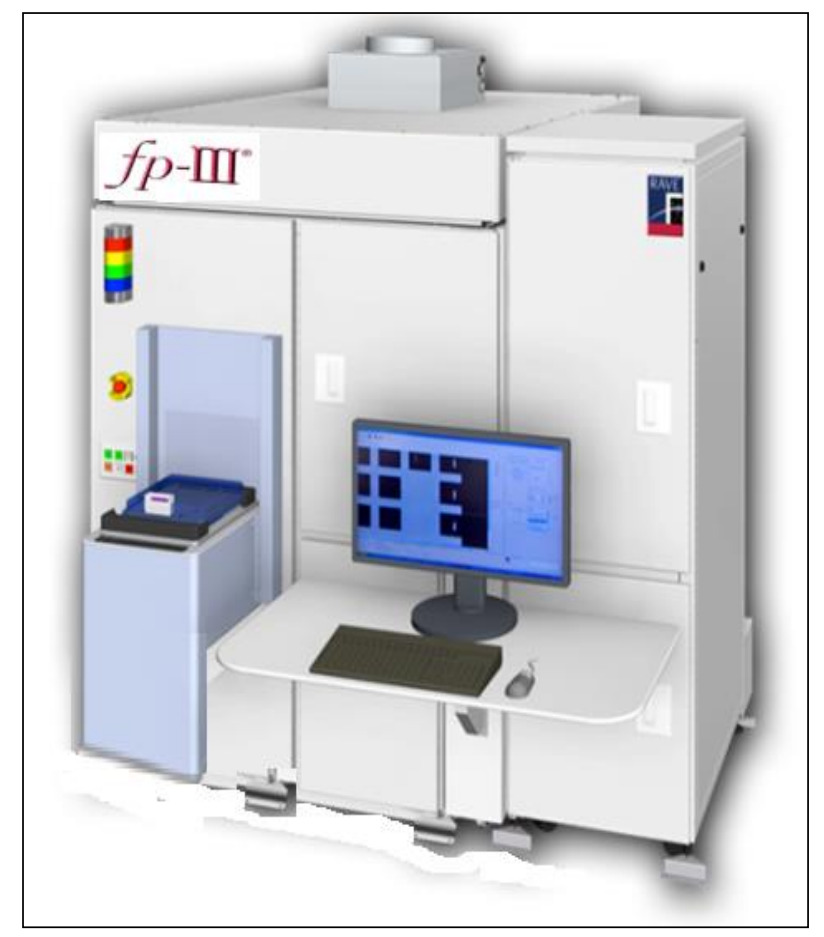

Figure 2, fp-III advanced laser photomask repair system 


\subsection{Sample for RESCAN EUV Metrology}

Figure 3 shows a summary of the test defect matrix pattern for the sample specifically made for the RESCAN apparatus. The sample's substrate was a silicon wafer cut to $2 \times 2 \mathrm{~cm}$ with a testing pattern in the center and two reference patterns in the upper corners. All repairs and AFM scans were performed on the center test pattern only which included a 4 x 4 array of die with identical patterns and defects. Each die (labelled 0-9 then A-F as shown in Figure 3) was then comprised of a 3 x 3 array of sub die (referred to by row and column number, for example row1 column 2, or R1C2). Each sub die was even further comprised of $3 \times 3$ um square cells, each of which could contain up to 50 defects. With this very large number of defect sites, most of the repairs were focused on one die (die B as seen in Figure 3), additional test repairs were performed in $\mathrm{R} 1 \mathrm{C} 3$ sub die of die $\mathrm{F}$ as well as some pre repair referencing metrology performed in dies $\mathrm{A}$ and $\mathrm{C}$.

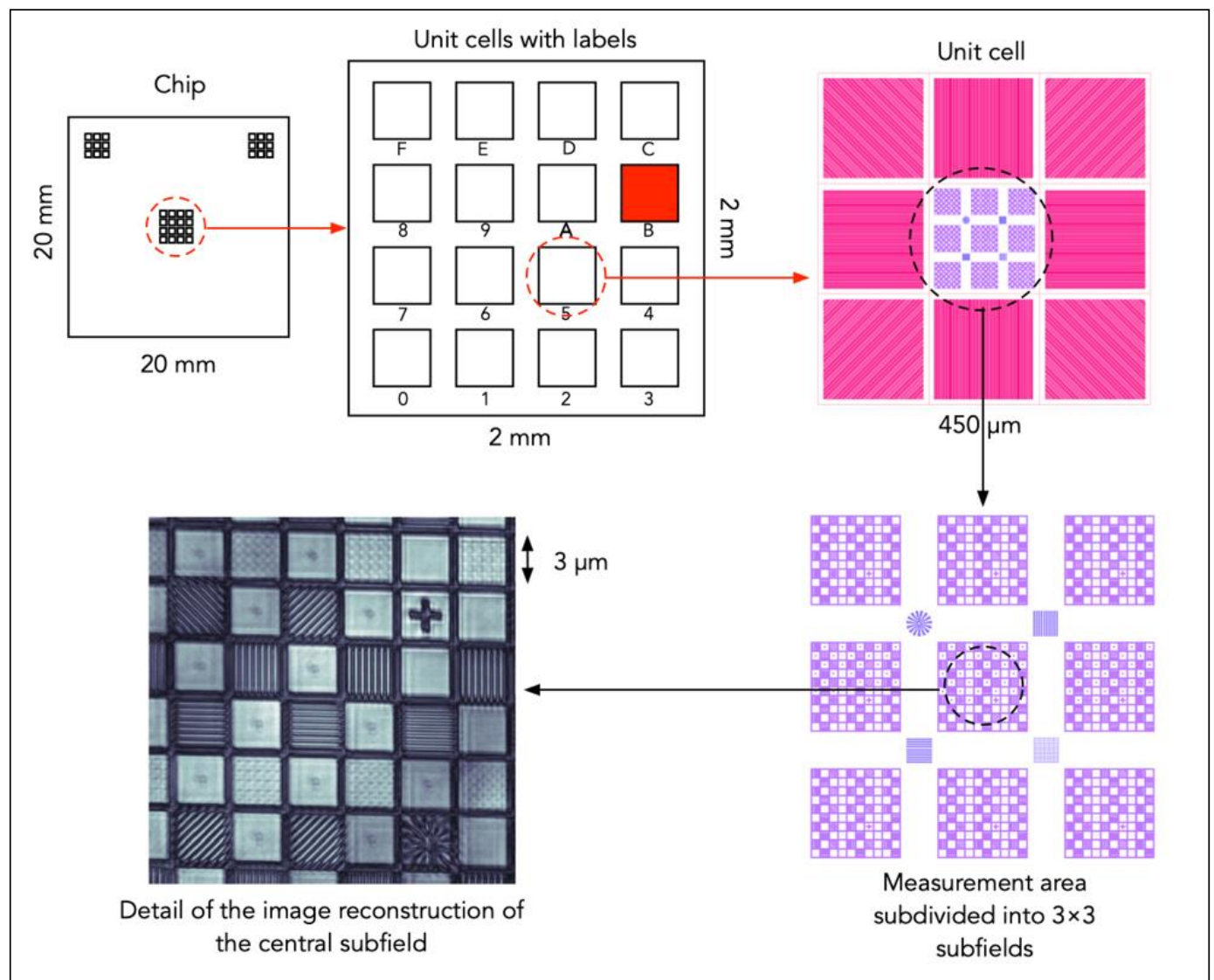

Figure 3, sample pattern layout for RESCAN EUV metrology

There were two different defect sizes (and aspects) evaluated and two different material compositions for the pin dots. These two materials were hydrogen silsesquioxane (HSQ) and carbon (C). The former material (HSQ) was the same material as the absorber material for all of the surrounding patterns and had a maximum height equal to the nominal absorber thickness of approximately $200 \mathrm{~nm}$. These were high aspect pin dot defects found only in row 3 of the $3 \times 3$ sub die matrix shown in Figure 3. Although the HSQ defects were processed, it should be noted that results for HSQ are not included here. 
The other defect material, the carbon (C) pin dots, came in two different sizes and aspect ratios as shown in Figure 4. In the first row of the $3 \times 3$ sub die were the high aspect pin dot defects as shown in the left panel of Figure 4 . As shown in the figure inset table, there were 91 of these $\mathrm{C}$ defects in die $\mathrm{B}$ with a $\mathrm{z}$ height range from 27.7 to $204.6 \mathrm{~nm}$ (mean 91.6) and a full-width half-max (FWHM) ranging from 131 to $306.5 \mathrm{~nm}$ (mean of 221.1). The low aspect c-pin dots in sub die row $2 \mathrm{had}$ a $\mathrm{z}$ height range from 12.5 to $19.7 \mathrm{~nm}$ (mean 13.8) and a FWHM range of 499.5 to $682.5 \mathrm{~nm}$ (mean 578.6). Calculating the aspect ratios from these numbers ( $\mathrm{AR}=\mathrm{z}$-height/FWHM) we get a range of 0.15 to 1.08 (mean 0.43 ) for the high aspect, and a range of 0.02 to 0.03 (mean 0.02) for the low aspect carbon pin dot defects.
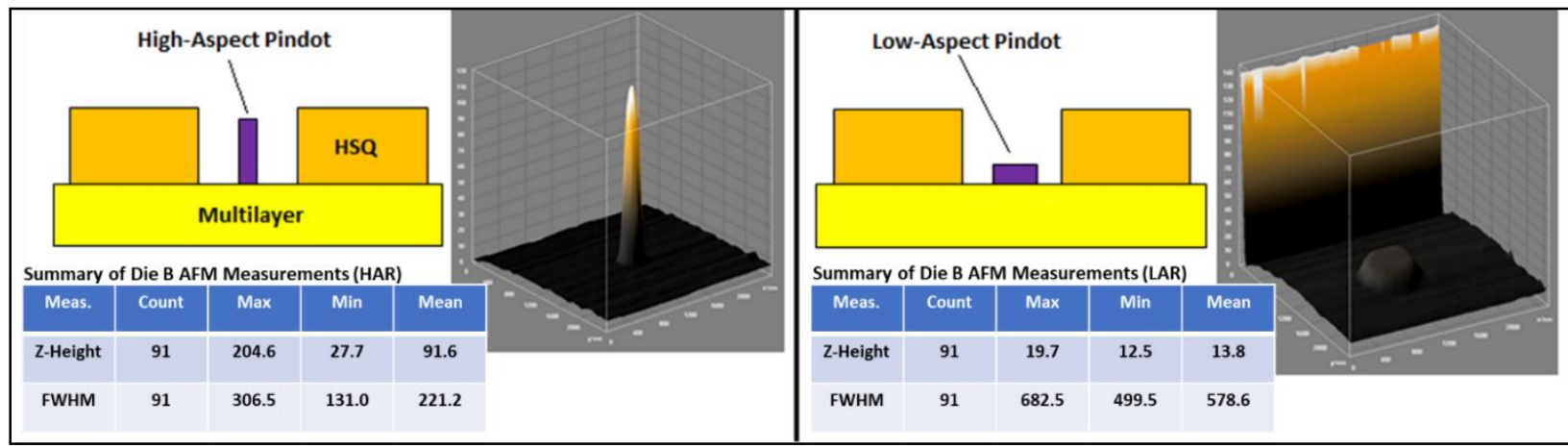

Figure 4. AFM inspection results for carbon pin dot defects in die B prior to laser test repairs. All units shown are in nanometers. A conceptual schematic, an example AFM scan, and raw AFM topographic measurements are shown for high aspect (left panel) and low aspect (right panel) defects.

In doing a large number of pre-repair AFM scans of die B, and other dies there were a couple of observations of interest that helped to define how the test procedure developed. The first observation was that although there was a large range in $\mathrm{C}$ defect sizes and aspect, there was some general groupings in the values. Thus, when the full factorial tests were defined for the laser processing parameters, the test sites were grouped so that their sizes were as close as possible to each other for that test. This meant that none of the tests were performed sequentially in placement on the sample but were scattered throughout the sub die row. It should be noted that for this work there are broad generalizations being made here in the defect sizes (high aspect versus low aspect defects). However, with further differentiation in these clusters, it may be possible to improve and clarify the analyses and modeling of the process data.

The second observation was that the variations in the high aspect carbon defects had a correlation to their location by, and within, each sub die. This feature of the test substrate was used to obtain comparable pre-data for those die where testing was performed, but no AFM pre-repair scans were collected.

Once the defect types were classified and grouped, the next consideration for test planning was the laser repair processes to be applied. Three different processes (parameters are not necessarily in order) with $2 \mathrm{x}$ different value ranges were evaluated. The first was the spot process where the laser beam is indexed to a location on the mask surface corresponding to the (DUV imaging) visual center of the defect. is the defect site is then exposed with a defined laser energy for some period of time and intensity. This process has three critical parameters for DOE tests (which for purposes here will be referred to as parameters $\mathrm{A}, \mathrm{B}$, and $\mathrm{C}$ ). The second process is the area process where the beam is moved over the mask surface in a defined region according to a programmed series of movement vectors in the XY plane 
(of the mask surface). This process was tested with five defined critical processing parameters (parameters A through E). The final, and third, process was a new process still currently in development. A systematic DOE was not performed with this new process. Instead, a best of breed set of parameter values, determined from prior demonstration repairs were used. For the third process, the primary objective was to determine what the removal efficiency was for the high aspect carbon pin dot defects and whether there were any detectable effects on multilayer EUV printability.

In total, there were at least six different laser processing parameters which were targeted as being critical in DOE testing. These parameters included spot overlap, number of laser pulses exposed to the surface, motion vector speed, focus offset, vector spacing, and mean laser pulse energy.

The measure looked at in the full factorial design of experiments (FFDOE) in this particular round of data analyses included the relative EUV phase shift impact on C pindot defects (post laser repair). For two of the FFDOE's a static spot process was used that necessitated test definition with 3 Laser Parameters (A, B, C) or in other words, 3 variable, and 3 states (i.e., minimum, median, and maximum values). There was one test with the high AR carbon pindots that used an area-scan process. This required a test with 5 Laser Parameters (A, B, C, D, E) or, 5 variables, and 2 states (maximum and minimum values only).

The amplitudes and phase shifts were measured from the difference between the pixel with the maximum absolute value and the mean value for a 50x50 pixel area centered in the test cell (see Figure 5). As previously mentioned, in the RESCAN images (one for EUV amplitude and one for relative phase shift) 1 pixel corresponds to approximately 32.5 $\mathrm{nm}$ on the sample. Thus, a 50x50 pixel sample area calculates as $1.625 \times 1.625$ microns in the $3 \times 3$ micron test cell.

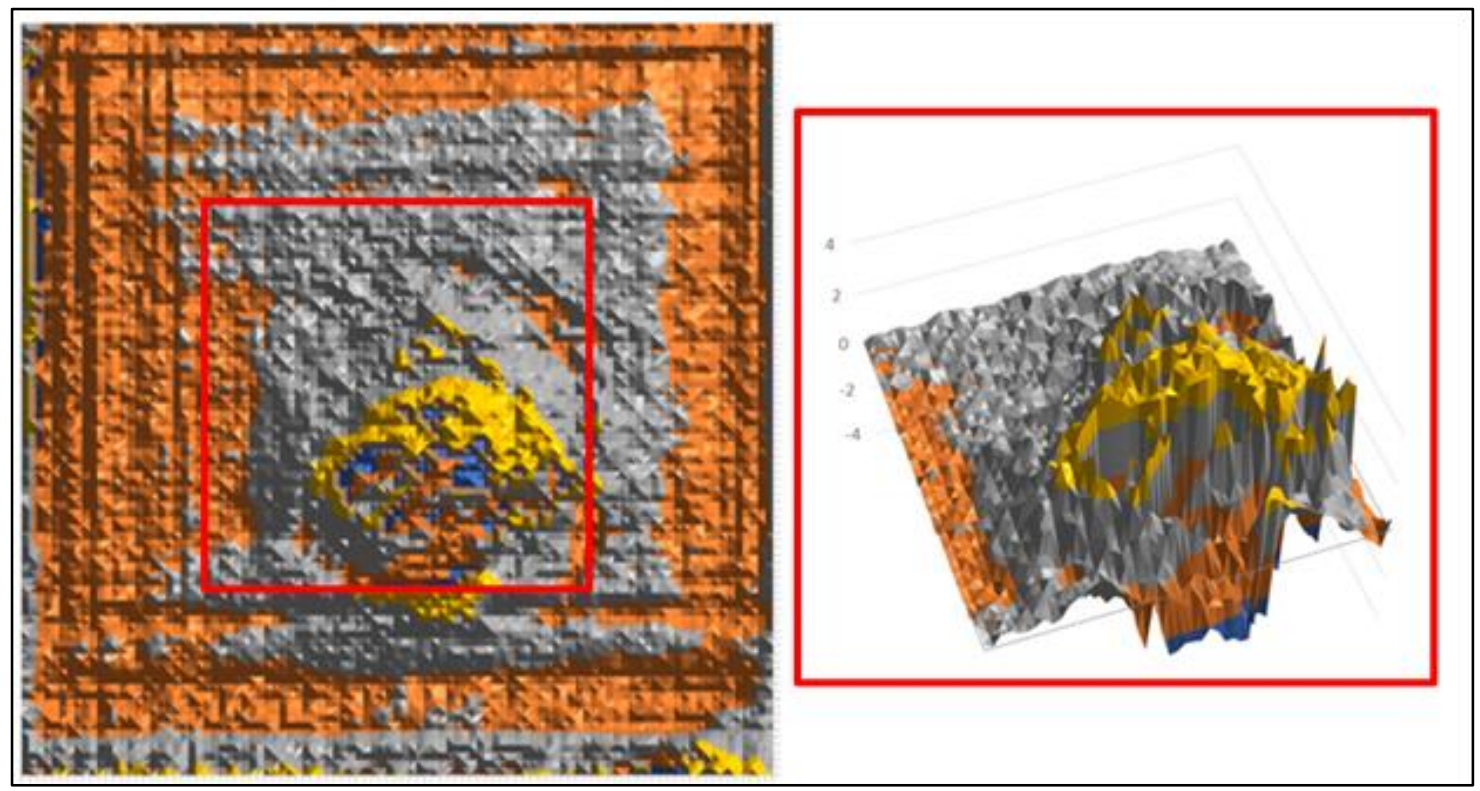

Figure 5. A 3D plot of an extreme example (to show the target defect) of RESCAN relative EUV phase data for a test cell. The red box represents the 50x50 pixel auto-sampled area (right panel) of a manually selected 100x100 pixel cropped test cell (left panel). 


\section{RESULTS}

\subsection{First Look at Raw Phase Data}

A first pass look at the raw results from the relative phase shift analyses is shown in left panel of Figure 6. In these plots, the data is just shown sequentially with no consideration to the laser parameters applied. One of the first observations in this plot is that there are no parameters that reached zero (or perfect) relative phase shift. This was because the data was given as the absolute maximum value for any point in the 50x50 pixel sampled box area and peak-to-peak background noise alone would assure a non-zero value. As shown in Figure 6, this background appears to be approximately 0.512 radians and a large number of data points are within this limit and thus indicate no net phase damage to the multilayer. Further, this indicates a large no-damage process domain for the laser processes analyzed (spot and area).

In the three stacked inset plots on the left side of Figure 6, the raw data sorted to the three tests examined are shown (spot process with low parameter A range, spot process with high parameter A range, and area process). In all of these plots, responses are shown indicative of one or more (possibly coupled) laser process parameters in phase shift results.

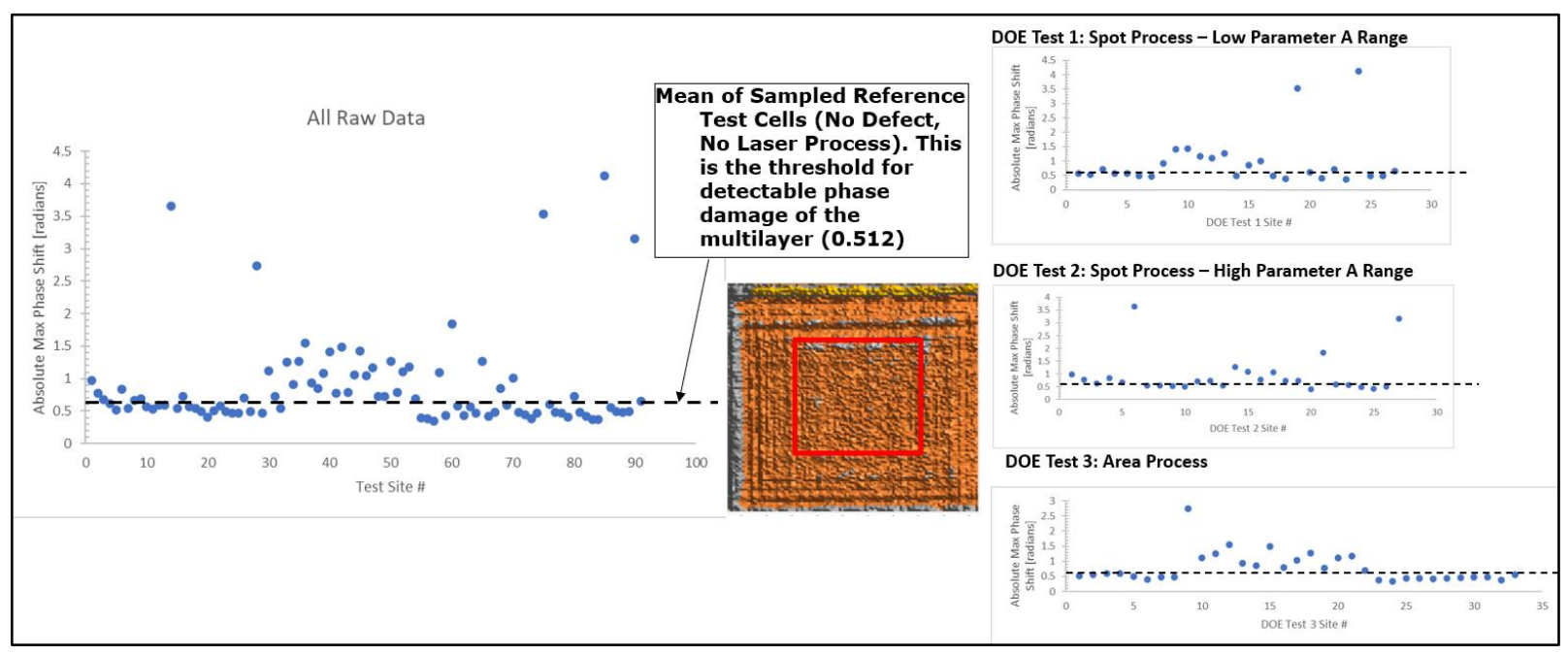

Figure 6. Initial examination of raw EUV phase data.

The DOE partial-pareto (hereafter referred to as just the pareto) for the spot process with low parameter A range (maximum to minimum) is given in Figure 7 (Test 1). In this chart, it is seen that at low parameter A range, the first order interaction of parameters $\mathrm{A}$ and $\mathrm{C}$ are the greatest influence with a positive interaction slope for parameter $\mathrm{C}$. Looking for any prevalence in the sign (+ or -) of the relative phase shift, it may also be seen that although the two polarities are close, there is a slight bias towards maximum phase damage with a negative phase shift. 


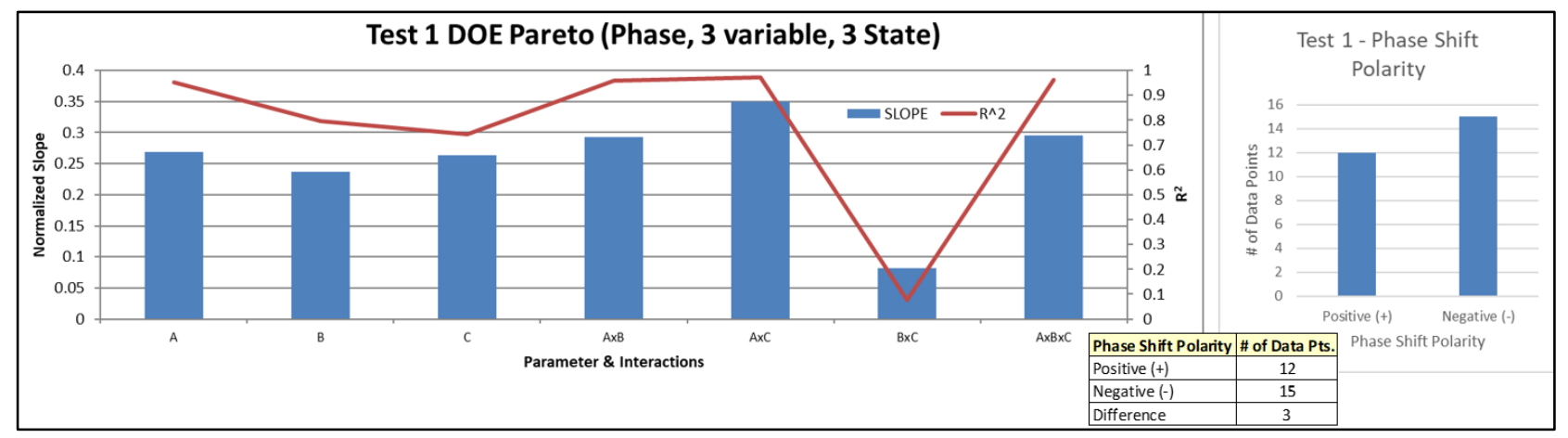

Figure 7. Results of 3 variable, 3 state full factorial DOE analysis of EUV-actinic phase shift of high-aspect carbon pindot laser repairs (Test 1). The test shown here is the spot process with low parameter A range.

In continuing an analysis of the spot process test at low parameter A range, the individual slope correlation plots are shown in Figure 8 (insets grouped to the right side of the figure) with a list of parameters ordered according to their significance in influencing the phase shift (inset, left side of Figure 8). Looking also at the $\mathrm{R}^{2}$ values to these linear correlations, it may be seen that this value appears to vary proportionately with the slope. This is most likely due to an increasing signal to noise ratio in the data as the slope increases. Another, potentially relevant, observation is that, in this process regime, the 3rd-order interaction of all 3 laser parameters is a close 2 nd (to the 1 st order interaction of parameters $\mathrm{A}$ and $\mathrm{C}$ ) in slope response impact.
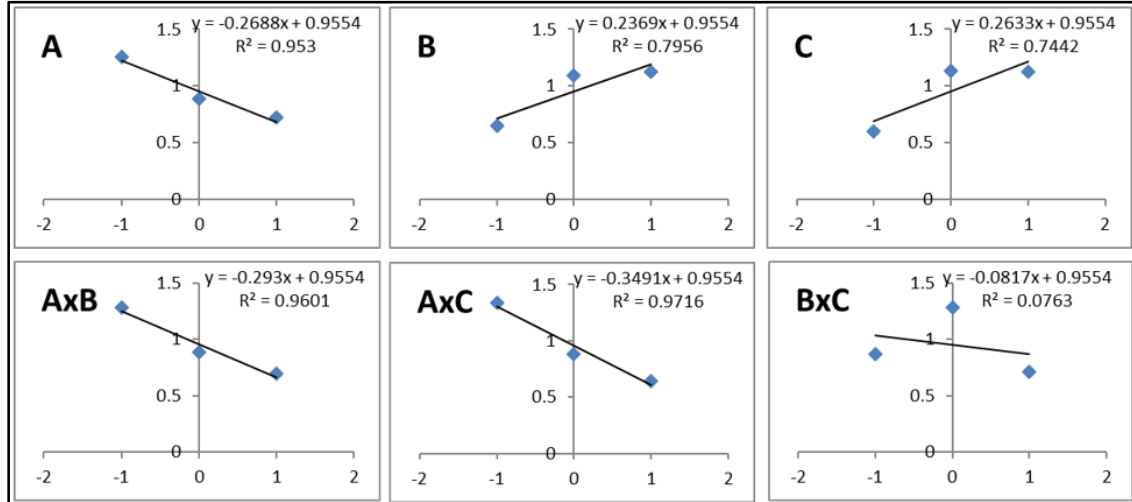

\begin{tabular}{|c|c|c|}
\hline Variable & $\begin{array}{l}\text { Mean } \\
\text { SLOPE }\end{array}$ & Polarity SLOPE \\
\hline AxC & 0.349 & -0.349 \\
\hline AxBXC & 0.295 & -0.295 \\
\hline AxB & 0.293 & -0.293 \\
A & 0.269 & -0.269 \\
\hline C & 0.263 & 0.263 \\
\hline B & 0.237 & 0.237 \\
BxC & 0.082 & -0.082 \\
\hline
\end{tabular}

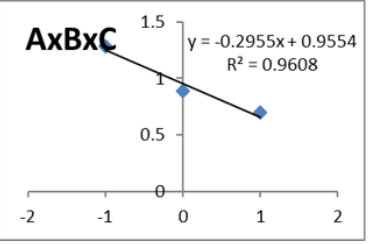

Figure 8. Continued phase analysis of DOE test of the spot process with low parameter A range (Test 1).

Figure 9 is the 3 variable, 3 state, DOE pareto for the test (designated Test 2) using the spot process with high parameter A range (maximum to minimum). It may be seen in considered examination of this chart that, with higher parameter $\mathrm{A}$ range, the first order interaction of parameters $\mathrm{A}$ and $\mathrm{C}$ are still the greatest influence. Also, it may be seen in the inset 
on the right side of the figure that, similar to the prior test (spot process with low A range), there is a slight bias towards maximum phase damage with a negative phase shift.

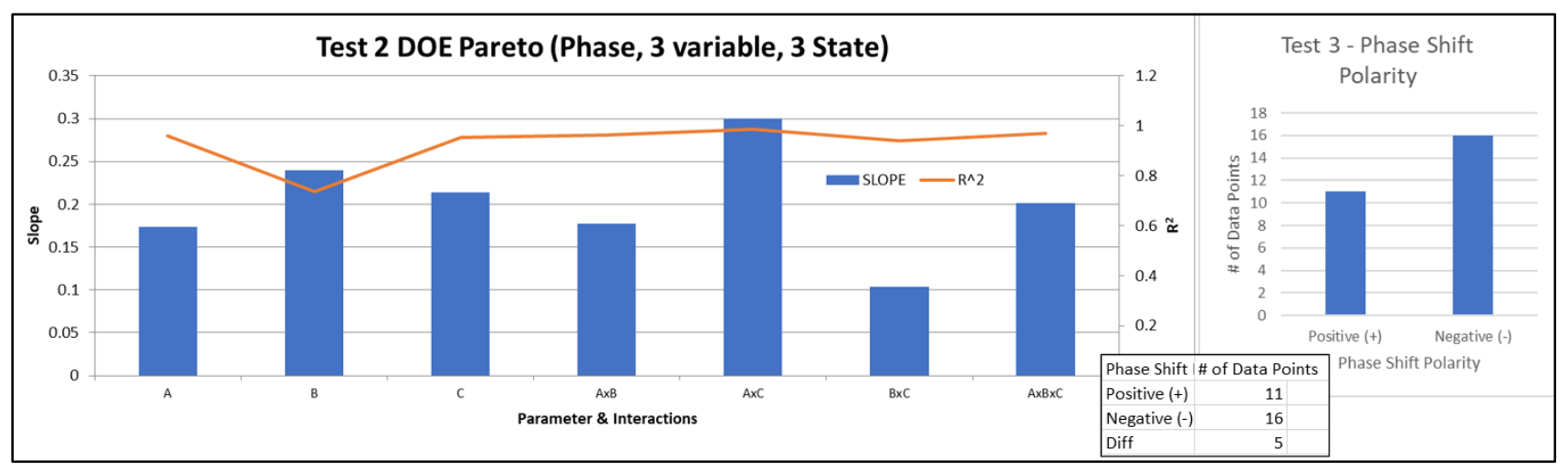

Figure 9. DOE pareto for test with spot process and high parameter A range (Test 2).

In Figure 10, a summary of the linear correlations for each parameter and parameter interaction (insets to the left side of the figure) with a table of these parameters and interactions ordered by slope (with the maximum magnitude at the top and then decreasing as one goes down the list). The $\mathrm{R}^{2}$ values for the linear correlation to the $\mathrm{BxC}$ interaction is significantly higher which makes logical sense with a physical understanding of these parameters. With a similar understanding, the increase in significance of parameter $\mathrm{B}$ and the observation that parameter $\mathrm{C}$ flips to a negative interaction slope (also explaining the slope flip in $\mathrm{AxC}$ interaction) may be logically revealing as to the process functions. In particular, the flip in parameter C's slope polarity may be related towards a hypothesized mechanism for enhanced multilayer subsurface damage.

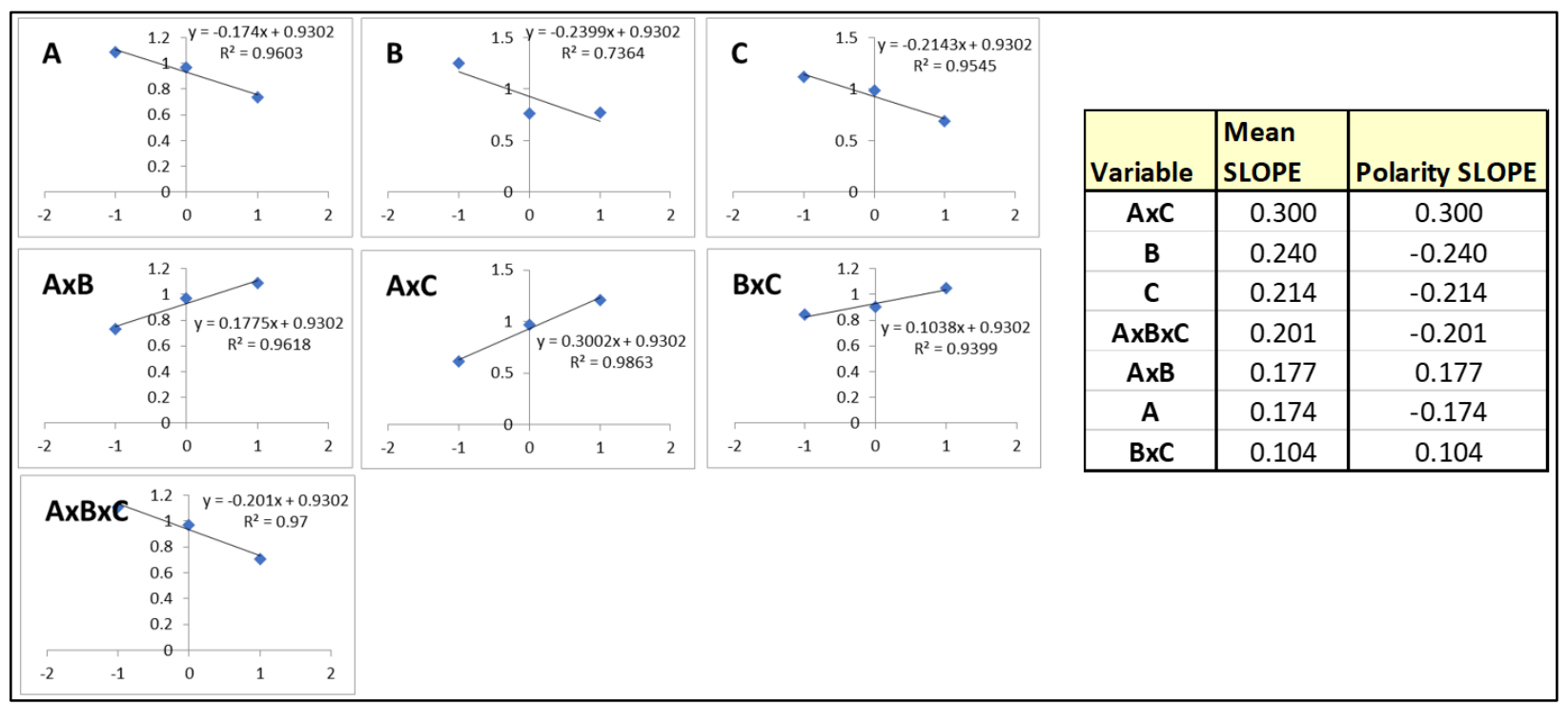

Figure 10. Linear correlations (left side insets) and parameter priority table (right inset) for Test 2 (spot process, high parameter A range). 
For the final DOE test examined here, is shown in Figure 11 as an area process requiring the characterization of 5 laser parameter (or 5 variable) test with 2 states (maximum and minimum values) only. This area process required significantly more input parameters, and to keep the required number of data points comparable to those required in the prior two tests, the number of states was reduced from 3 to 2 .

The most obvious observation from the graph in the left inset of Figure 11 is that the first-order interaction of $\mathrm{AxB}$ is significantly a greater influence on the phase shift results than any other parameter or parameter interaction (a total of 4levels of parameter interactions were examined). An understanding of the physical process, and the experience of the state-of-the-art practitioner, is confirmed in that this predominate influence is with the parameter interaction AxB.

It may also be seen in the inset on the right-hand side of Figure 11 that the difference between maximum negative and positive phase shift damage is significantly greater for the area process than the spot process. This has been a recurring observation for all three tests reviewed here and (it is speculated) may be due to how the first order nature of (potentially dose cumulative) subsurface multilayer damage may alter the phase shift of reflected EUV light.

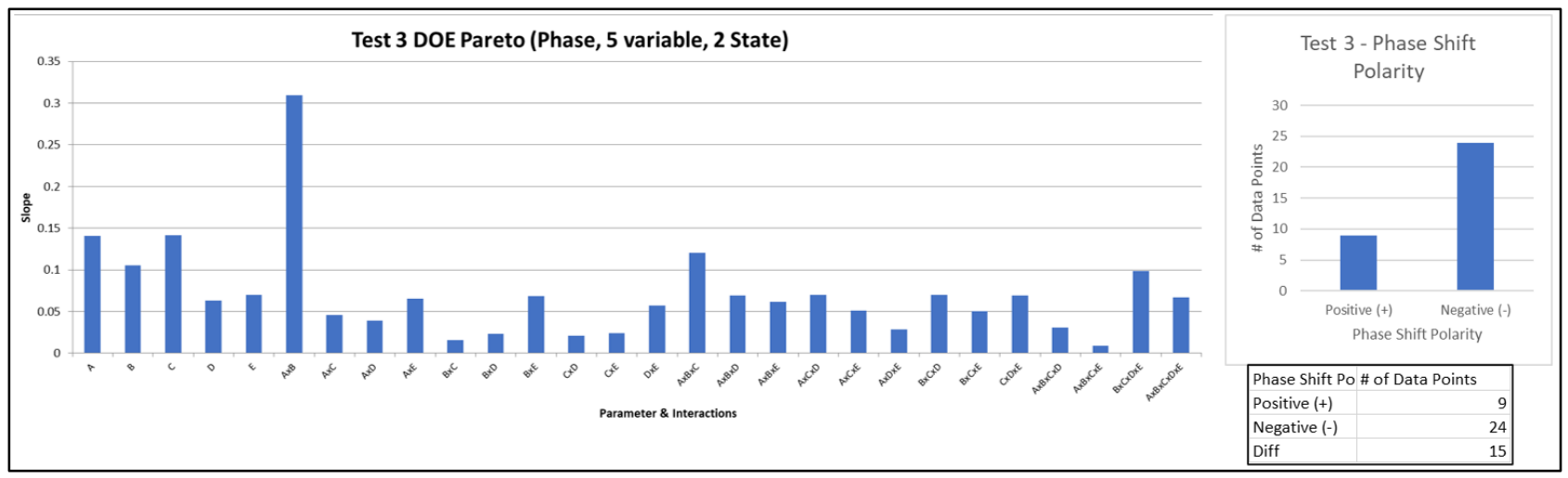

Figure 11. DOE pareto for test with area process.

As seen in Figure 12, are the slope correlations for Test 3 area process. Since there were only two states in this test, the $\mathrm{R}^{2}$ parameter, which indicated the degree of correlation of the data point means to the linear function which yielded the slope value, was irrelevant.

In this examination of the data, we focus on the parameters A and B since their first order interaction so clearly predominated over any other tested factor. One may see that the polarity of the slopes of parameter A and B are opposite to each other, which makes sense to the knowledgeable process engineer. However, looking at the particular polarities one sees that the optimal process (yielding minimal phase shift damage on the multilayer) would be to maximize parameter A (negative slope) while minimizing parameter B (positive slope). This is the opposite of what process understanding and experience would recommend. This was resolved in a follow-up analysis (not shown, all results here are post repair) where the difference in absolute maximum phase shifts before and after laser repair where the polarities of these parameters were both reversed. 


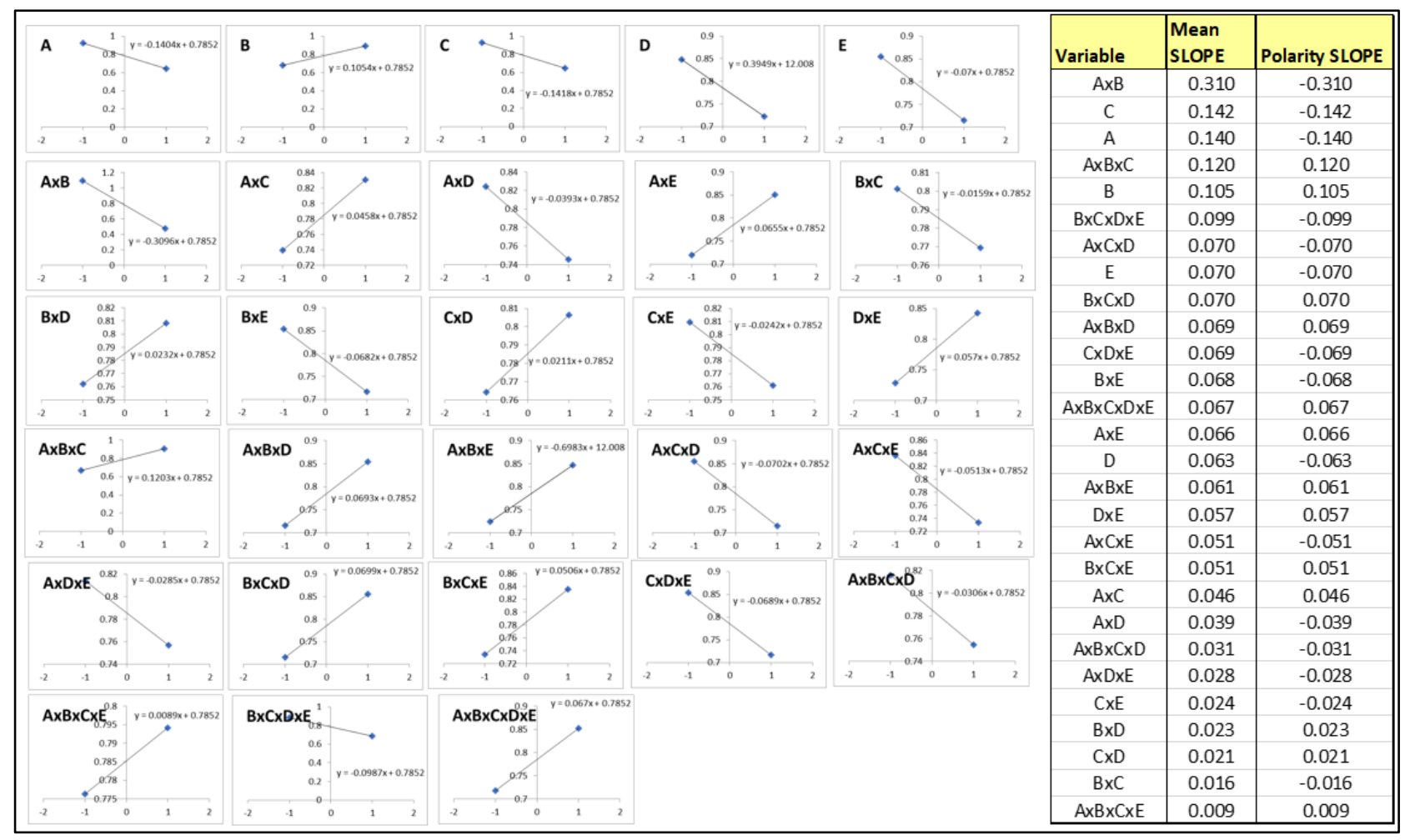

Figure 12. Linear slope correlations (left side insets) and parameter priority table (right inset) for Test 3 (area process).

\section{CONCLUSIONS}

A number of observations from Full-Factorial Design of Experiments (FFDOE) tests and analyses of laser induced phase shift on EUV multilayer indicated potential process improvements for spot and area processes. For the spot process at low parameter $\mathrm{A}$ range, the first order interaction of parameters $\mathrm{A}$ and $\mathrm{C}$ are the greatest influence with a positive interaction slope for parameter $\mathrm{C}$. With higher parameter $\mathrm{A}$ range in the spot process, the first order interaction of parameters $\mathrm{A}$ and $\mathrm{C}$ are still the greatest influence, however, parameter B increases in significance and parameter C flips to a negative interaction slope. The polarities (whether negative or positive) phase shifts for the test pindot defects post laser repair were examined, and there appeared to be a slight bias towards maximum phase damage with a negative phase shift.

For the area process, the AxB first order parameter interaction is clearly the greatest influence in the area process. The difference between maximum negative and positive phase shift damage is significantly greater for the area process than the spot process. Further, looking at the slopes of A and B, it would appear to be optimal to increase A and decrease B which was counter-intuitive to prior process experience. This was resolved in further analyses (not show) of the differential (post-pre laser repair) maximum absolute phase shift which reversed the polarities of both parameters (A and B). 
The observed significant parameters in EUV phase shift correspond to those seen in FFDOE testing of AFM measured volume percent change in the high aspect $\mathrm{C}$ pindot defects. 1 It is seen in the figure in the first, second, and third tier (the tiers corresponding to the priority in their effect on the measurement being analyzed) parameters and parameter interactions are compared to the prior publication. ${ }^{1}$ The first two tests, tests 1 and 2 are compared to the AFM measurements of percent volume change of the same pindots repaired with laser spot process (only these tests are compared since test 3 was an area process). In this comparison, it may be seen that all of the first-tier results are the same $(\mathrm{AxC})$ for both tests $(1$ and 2$)$. The second-tier results correspond for Test 1 (AxBxC), but for Test 2 the single parameter $\mathrm{C}$ was bumped up from third to 2 nd tier. In the third tier, neither Test 1 nor 2 correspond to the results in the prior study, possibly due to this tier falling below the pareto principle limit in significance.

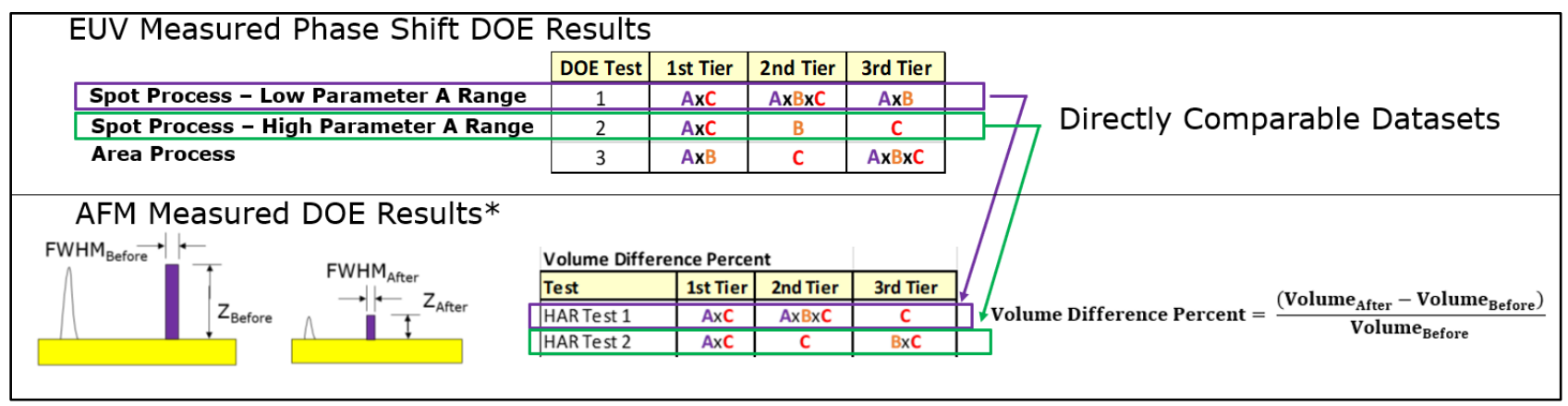

Figure 13. A comparison of a summary of results from this (top panel) to the prior publication ${ }^{1}$ (bottom panel). For comparable tests, the pindot z-height and phase results had a high degree of correspondence in the 1st and 2nd tier parameters and parameter interactions.

Overall, this comparison confirms the reality of these results and the approaches used to perform these analyses. These results will aid in furthering the development of a femtosecond laser process that will quickly and efficiently repair and clean EUV photomasks, in blank, and patterned regions well into sub-resolution technology nodes in the future.

\section{REFERENCES}

[1] Tod Robinson, Jeff LeClaire, Iacopo Mochi, Ricarda Maria Nebling, Yasin Ekinci, Dimitrios Kazazis, "Laser repair and clean of extreme ultraviolet lithography photomasks," Proc. SPIE 11518, Photomask Technology 2020, 1151809 (13 October 2020); https://doi.org/10.1117/12.2573084

[2] Tod Robinson, Jeff LeClaire, "Advanced laser repair of EUV photomasks," Proc. SPIE 11178, Photomask Japan 2019: XXVI Symposium on Photomask and Next-Generation Lithography Mask Technology, 111780K (27 June 2019); https://doi.org/10.1117/12.2534759.

[3] Iacopo Mochi \& Yasin Ekinci (2019) Lensless EUV Lithography and Imaging, Synchrotron Radiation News, 32:4, 22-27, DOI: 10.1080/08940886.2019.1634433. 
[4] Thibault P, Dierolf M, Bunk O, Menzel A, Pfeiffer F. Probe retrieval in ptychographic coherent diffractive imaging. Ultramicroscopy. 2009;109(4):338-343. doi:10.1016/j.ultramic.2008.12.011.

[5] Iacopo Mochi, Sara Fernandez, Ricarda Nebling, Uldis Locans, Rajendran Rajeev, Atoosa Dejkameh, Dimitrios Kazazis, Li-Ting Tseng, Serhiy Danylyuk, Larissa Juschkin, Yasin Ekinci, "Quantitative characterization of absorber and phase defects on EUV reticles using coherent diffraction imaging," J. Micro/Nanolith. MEMS MOEMS 19(1) 014002 (30 January 2020) https://doi.org/10.1117/1.JMM.19.1.014002. 